\title{
PROSODIC FEATURES OF VERY SHORT UTTERANCES IN DIALOGUE
}

\author{
Jens Edlund \\ Mattias Heldner \\ Antoine Pelcé
}

\section{Introduction}

A large number of vocalizations in everyday conversation are traditionally not regarded as part of the information exchange. Examples include confirmations such as yeah and $o k$ as well as traditionally non-lexical items, such as $u h$-huh, um, and $\mathrm{hmm}$. Vocalizations like these have been grouped in different constellations and called different names, for example backchannels (i.e. back-channel activity, Yngve, 1970), continuers (Schegloff, 1982), feedback and grunts, and attempts at formalizing their function and meaning have been made (e.g. Ward, 2004). We follow (Ward \& Tsukahara, 2000), who argue that the term backchannel feedback is relatively neutral, and henceforth use the term backchannel.

The working definitions of these overlapping concepts, however, are imprecise, and different labeling schemes treat them quite differently, often with dubious inter-annotator agreement numbers as a result (e.g. Hardy, et al., 2003). The schemes are also often complex. A typical complication occurs with the distinction between an answer of some type, say "I agree", and a backchannel signaling understanding, say "mhm". The distinction between the two relies heavily on third-party judgments of the speakers' intentions, such as "was a response required or optional here?" or "was this acknowledgment unsolicited or prompted by a request?". In some coding schemes, the distinction is based on lexical context. SWBD-DAMSL, for example, states that "yeah" belongs to the category AA (AGREEMENT-ACCEPT) if followed by additional verbal evidence, such as "me too", while it is B (BACKCHANNEL, or ACKNOWLEDGE) if uttered in isolation (Jurafsky, Shriberg, \& Biasca, 1997).

In spite of difficulties involved in defining these phenomena, there is little controversy surrounding the utility of modeling them. They behave differently from other utterances, and so are interesting both for models of human conversation and for spoken dialogue systems aiming at more human-like behavior. Commonly reported characteristics include that they can be spoken in overlap without disrupting the original speaker (hence the term backchannel), they 
are relatively brief, and inspection of their constituent tokens (e.g. Ward, 2004) reveals that they contain almost exclusively voiced sounds.

Inspired by others, for example Shriberg et al. (1998), who state that "duration was expected to be a good cue for discriminating STATEMENTS and QUESTIONS from dialogue acts functioning to manage the dialogue (e.g. BACKCHANNELS)", we propose to use an intermediate, auxiliary unit - the very short utterance (VSU) - which is defined operationally and is automatically extractable from recorded dialogues. VSUs are intended to capture a large proportion of interactional dialogue phenomena such as those commonly referred to as backchannels, feedback, continuers, et cetera, at zero manual effort.

Note that we are not describing a backchannel classifier. We tentatively define VSUs in terms of prosodic characteristics commonly associated with backchannels, specifically using thresholds for duration and ratio of voiced versus unvoiced frames. VSUs are then automatically extracted from a corpus of spontaneous two-party dialogues. The corpus is also manually labeled for backchannel-likeness, and the manual labels are compared with the VSUs. Finally, we present visualizations of pitch contours for VSUs and NONVSUs.

\section{Methods}

\subsection{Materials}

We used speech material from the Swedish Map Task Corpus (Helgason, 2006), designed as a Swedish counterpart to the HCRC Map Task Corpus. The map task is a cooperative task involving two speakers, intended to elicit natural spontaneous dialogues. Each of two speakers has one map which the other speaker cannot see. One of the speakers, the instruction giver, has a route marked on his or her map. The other speaker, the instruction follower, has no such route. The two maps are not identical and the subjects are explicitly told that the maps differ, but not how. The task is to collaborate in order to reproduce the giver's route on the follower's map. Eight speakers, five females and three males, are represented in the corpus. The speakers formed four pairs, three female-male pairs and one female-female pair. Each speaker acted as instruction giver and follower at least once, and no speaker occurred in more than one pair. The corpus includes ten such dialogues, the total duration of which is approximately 2 hours and 18 minutes. The dialogues were recorded in an anechoic room, using close-talking microphones, with the subjects facing away from each other, and with acceptable acoustic separation of the speaker channels.

\subsection{Procedures}

The following procedures were involved in the study; each step is described in detail in the following subsections: 
- Automatic segmentation of verbal production into talkspurts; talkspurts are subsequently treated as atomic units.

- Manual labeling of the talkspurts, using a simple annotation scheme, as either backchannel-like (Low propositional content) or not backchannellike (NONLOW propositional content).

- Automatic classification of the talkspurts into VSUs and NONVSUs, using duration and ratio of voiced frames.

- Quantitative and visual comparison of manual and automatic labels.

- Extraction and visualization of F0 patterns - both in VSUs and in interlocutor speech immediately preceding each VSU.

\subsubsection{Segmentation into talkspurts}

Although the concept of utterance makes for a useful unit, segmentation into utterances typically relies on higher-level linguistic knowledge, which we attempt to avoid here. Instead, we propose as our candidate unit a more convenient and less ambiguously defined segment, namely the talkspurt defined by Norwine \& Murphy: "A talkspurt is speech by one party, including her pauses, which is preceded and followed, with or without intervening pauses, by speech of the other party perceptible to the one producing the talkspurt" (Norwine \& Murphy, 1938). We note that this definition differs from that used by Brady (1968), in which a talkspurt is a sequence of speech activity flanked by silences in one speaker's channel. Brady's definition has been used by ourselves in previous work (e.g. Edlund \& Heldner, 2005; Laskowski, Edlund, \& Heldner, 2008), but Norwine \& Murphy's concept is better suited for our current purposes, and we adopt their definition in what follows. Note that a talkspurt, as used here, may contain pauses and overlapping speech; a talkspurt from one participant is said to end only when solitary speech from the other participant is encountered. Any initial or final silence is removed from the talkspurt proper, as illustrated in Figure 1.

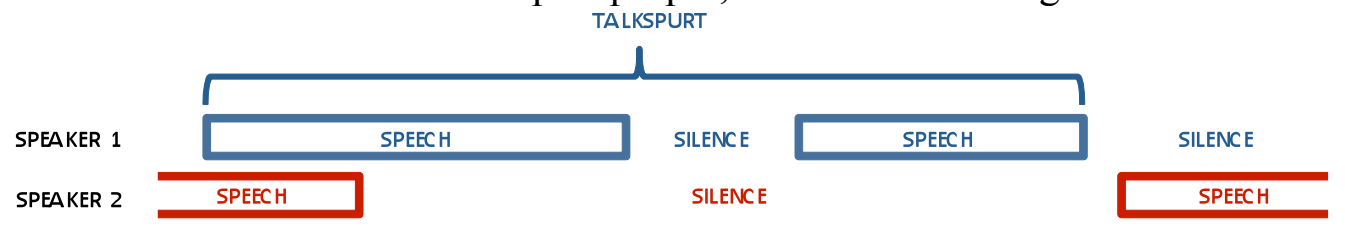

Figure 1. Schematic illustration of a talkspurt as used in the current work.

The VADER voice activity detector from the CMU Sphinx Project (http://cmusphinx.sourceforge.net/) was used to perform speech activity detection individually for each speaker. As a result, each instant for each speaker was labeled as either SPEECH or SILENCE. The combined information from both speakers is sufficient to extract all talkspurts. 


\subsubsection{Manual annotation of talkspurt backchannel-likeness}

In order to compare the proposed VSU unit with existing conceptualizations of backchannels, we manually annotated all talkspurts in the Swedish Map Task Corpus. The annotation scheme used is based primarily on the amount of propositional content, as backchannels are generally considered to be related to interaction control and hence to be relatively devoid of propositional content. Additionally, the scheme: (a) is based on non-prosodic features; (b) is independent of linguistic theory; and (c) yields good cross-annotator agreement among untrained annotators It consists of three mutually exclusive labels: HIGH (content), LOW (content), and Misc(ellaneous). Low designates the backchannel-like talkspurts that we were out to capture. We deliberately opted not to use a more comprehensive scheme, such as that proposed by Ward \& Tsukahara (2000), since the latter requires annotators to make judgments based on the context of the talkspurt. Another reason for adopting a simpler scheme was the need for annotation to be fast and accurate on the first attempt.

Our scheme asked annotators to apply ordered questions which, if answered in the positive, lead to a classification of HIGH or MISC, respectively. If both were answered in the negative, the talkspurt was categorized as Low. The instructions were phrased as follows:

1. Does the talkspurt contain high-content elements: objects (e.g. nouns (excluding pronouns) or proper names), attributes (e.g. adjectives) or actions (e.g. verbs, not including modal verbs and codas)? If so, label as HigH.

2. Does the talkspurt seem to be the result of a technical error when extracting the talkspurts; or does it consist of a disfluency, breathing, coughing, laughter, etc.? If so, label as MisC.

3. Otherwise label as Low.

The annotation scheme was applied to all talkspurts by two annotators working independently. Once annotation was begun, the annotators did not discuss the scheme until all talkspurts were annotated.

\subsubsection{VSUs}

The objective of automatically defining VSUs draws on the observation that backchannel-like phenomena are limited in duration and tend to contain mainly voiced speech sounds. Thus, we extracted two parameters from each talkspurt: its DURATION, from start to finish (see Figure 1); and its voicing ratio (VOICINGRATIO) - the proportion of frames in the talkspurt marked as voiced by YIN, an automatic pitch extractor (de Cheveigné \& Kawahara, 2002). A talkspurt was classified as a VSU if both of the following were true: 
1. the talkspurt's DURATION was shorter than a given threshold, and

2. the talkspurt's VOICINGRATIO exceeded a given threshold.

Finding optimal thresholds for Duration and VoicingRatio is a task for future work. For the present study, we tentatively set them by testing each combination of observed VoICINGRATIO and DuRATION, and selecting the threshold pair resulting in the smallest sum of false positives and false negatives when compared to the manual backchannel-likeness annotation. Note, however, that the manual Low annotation is a crude approximation of the set of backchannel-like activity that we aim to capture.

\subsubsection{Extracting F0 patterns}

For each talkspurt, F0 estimates of the last $500 \mathrm{~ms}$ of speech were computed using YIN (de Cheveigné \& Kawahara, 2002). These were transformed from Hertz to semitones to make the pitch excursions of men and women more comparable. The data was subsequently smoothed using a median filter over $910 \mathrm{~ms}$ frames to eliminate outlier errors. The resulting contours of smoothed F0 estimates were shifted vertically such that the median of the first three voiced frames in each contour fell on the mid-point of the y-axis; they were also right-aligned horizontally such that the end of the talkspurt lay on the right edge of the resulting diagram. By plotting the contours with partially transparent dots, the visualizations give an indication of the distribution of different patterns, with darker bands for concentrations of patterns and vice versa. This visualization, which we refer to as bitmap clustering, has also been used in (Heldner, Edlund, Laskowski, \& Pelcé, forthcoming).

In addition, for each talkspurt, we extracted the F0 estimates of the last $1000 \mathrm{~ms}$ in the interlocutor's channel, immediately preceding the talkspurt in question. We note that in some cases, this interval would consist mainly, or even entirely, of silence. These were again semitone transformed and median filtered, and then Z-normalized for each speaker along the vertical octave axis; the mean and standard deviation was computed using all voiced speech available from the interlocutor. Along the horizontal time axis, no shifting was done as the segments were all of the same length. These contours were also visualized using bitmap clustering.

\section{Results and discussion}

Segmentation yielded a total of 3054 talkspurts. In this section, we report on the per-talkspurt distribution of manually annotated propositional content, LOW and NonLow, as well as on that of the automatically generated VSU/non-VSU labels; the bivariate distribution suggests that, at the talkspurt level, degree of propositional content is strongly correlated with our operations VSU definition. 
We show how the parameters used to define VSUs, DURATION and VOICINGRATIO, are distributed across talkspurt types, and present the distributions of talkspurt-terminating pitch contours, both inside each talkspurt type and inside the interlocutor's most recent talkspurt. Together, these results show that VSUs represent a consistent group of talkspurts with characteristics not present in NONVSUs.

\subsection{Manual annotation}

Both annotators labeled all 3054 utterances in one sweep, in less than 4 hours. The agreement between the two annotators was high, with a kappa of 0.887 for all three categories LOW, HigH and MisC. Agreement increases to 0.895 when HiGH and MISC are collapsed, yielding the binary distinction (LOW/NONLOW) of greatest interest here. Table 1 shows the inter-annotator agreement matrix.

Table 1. Manual annotation confusion matrix.

\begin{tabular}{rlrrrr}
\hline & & \multicolumn{4}{c}{ Rater 1 } \\
& & Low & HigH & MisC & Total \\
\hline \multirow{4}{*}{ Rater 2 } & LOW & $\mathbf{9 0 3}$ & 93 & 15 & 1011 \\
& HIGH & 10 & $\mathbf{1 6 5 6}$ & 12 & 1678 \\
& MisC & 21 & 44 & $\mathbf{3 0 0}$ & 365 \\
& Total & 934 & 1793 & 327 & $\mathbf{3 0 5 4}$ \\
\hline
\end{tabular}

Further analysis showed that the disagreement in the Low category - the category of interest here - is largely due to the first annotator using a wider concept for the category. The second annotator's Low category is a subset of that of the first, and there are very few instances (31) where the second annotator has used Low and the first has not.

\subsection{DURATION and VOICINGRATIO in relation to manual annotation}

The distributions of DURATION for talkspurts labeled as LOW, HIGH and Misc by one annotator (the first annotator is used consistently in this section; results for the second annotator are similar) are shown in Figure 2. It can be seen that the mode for Low talkspurt durations lies in the $300-400 \mathrm{~ms}$ range, and that there are very few Low talkspurts whose duration falls below 200ms. For HigH talkspurt durations, the mode is clearly above $1000 \mathrm{~ms}$; there are very few HiGH talkspurt durations below 400ms. Furthermore, talkspurts can be quite long, well above 10 seconds. The mode for MISC is similar to that of LOW, around $200-300 \mathrm{~ms}$, suggesting that LOW and MISC may not be discriminated using DURATION alone. 

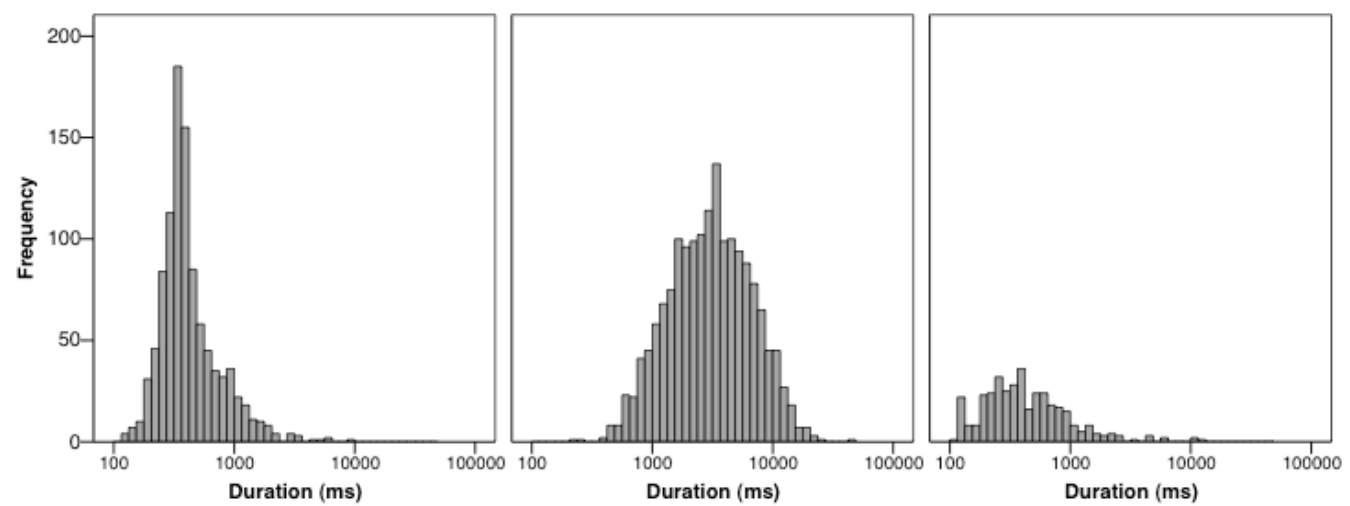

Figure 2. Distributions of DURATION of talkspurts annotated as LOW, HIGH and MISC (from left to right) by the one annotator. The $\mathrm{x}$-axis is in $\mathrm{ms}$ on a logarithmic scale.

Figure 3 shows the distributions of VOICINGRATIO for each of LOW, HIGH and MisC talkspurts. As can be seen, LOW talkspurt VOICINGRATIO is severely skewed towards unity; a large number of these talkspurts consists of at least $80 \%$ voiced frames, and almost no Low talkspurt is entirely unvoiced. HIGH talkspurt VOICINGRATIO shows a clear mode at $50-60 \%$ voiced frames, with very few fully voiced or fully unvoiced talkspurts. It also appears that MISC has one weak mode similar to that found in $\mathrm{HIGH}$, and one consisting of a very high spike in the $0-5 \%$ VOICINGRATIO bin, possibly corresponding to breathing, throat clearing, etc.
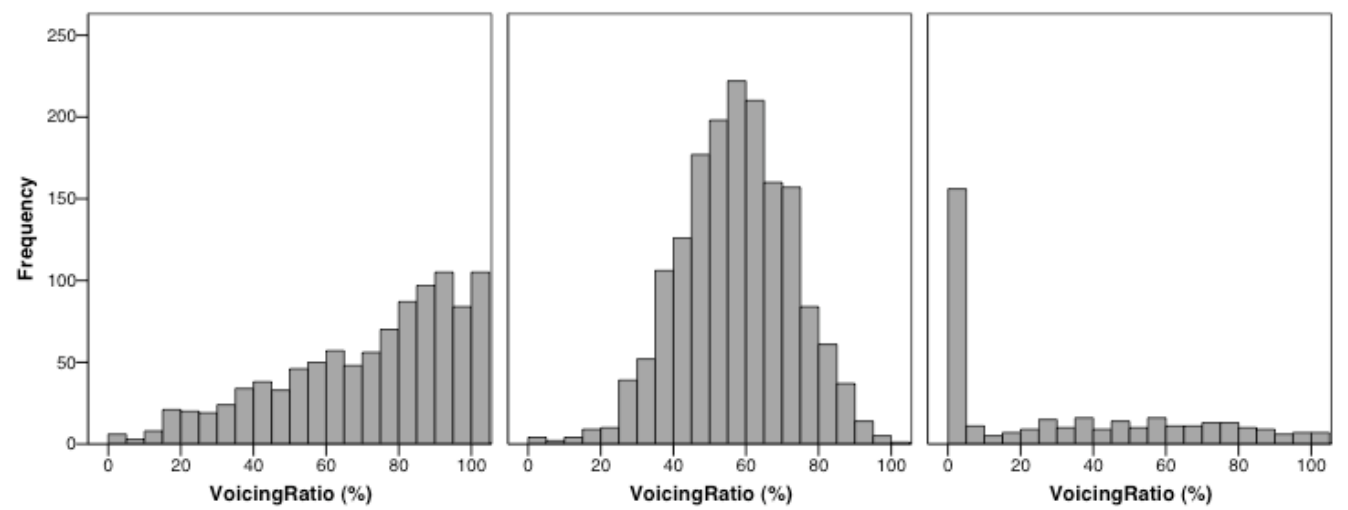

Figure 3. Distributions of VOICINGRATIO of talkspurts annotated as LOW, HIGH and MISC, respectively, by one annotator. 


\subsection{VSU classification in relation to manual annotation}

The distributions in Figures 2 and 3 suggest that discrimination of Low talkspurts may indeed be possible using VoICINGRATIO and DuRATION. Figure 4 shows ROC (Receiver Operating Characteristic) curves describing how well DURATION and VOICING, respectively, discriminate among talkspurt types; manual annotation of LOW by one annotator was used as the reference. Both curves are statistically significant deviations from no discrimination (the diagonal line), with an asymptotic significance of $<0.001$. However, VOICINGRATIO alone is a weaker predictor than DURATION alone (the areas above the line of no discrimination are 0.728 and 0.884 , respectively). This is also the case with respect to the asymptotic $95 \%$ confidence interval, where the upper bound of the interval for VOICINGRATIO is below the lower bound of the interval for DURATION.

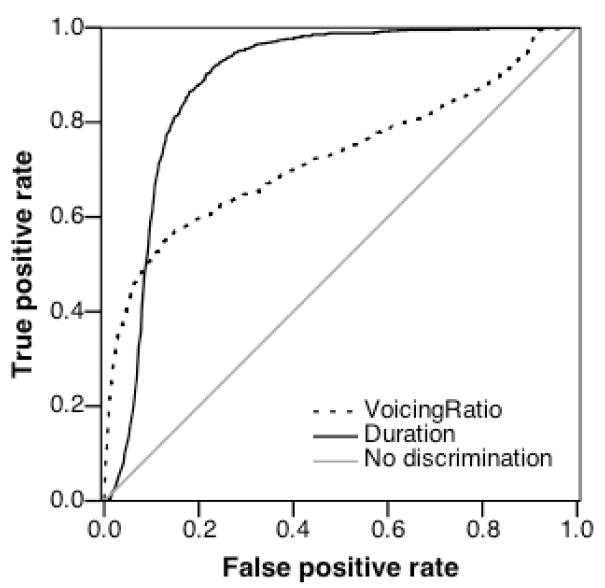

Figure 4. ROC curves for LOW/NONLOW classification using a continuum of VOICINGRATIO and DURATION thresholds.

We note that the ROC curve for DURATION alone shows a significant false positive rate of approximately 0.1 before it achieves a true positive rate of 0.5 ; thereafter, the curve asymptotically approaches a true positive rate of unity. Inspection of the thresholds resulting in this curve reveals that this is because the proportion of talkspurts judged as LOW increases with decreasing DURATION, but that in the end there are a certain number of NONLOW with very short DURATION - mainly breathing, laughter, coughs, and disfluencies - and these cannot be separated from Low talkspurts by means of duration.

In contrast, the VOICINGRATIO ROC curve incurs much fewer false positives before reaching a true positive rate of 0.5 , but does not attain a true positive rate near unity until the false positive rate exceeds 0.95 . Closer inspection suggests that this is because almost all talkspurts with a very high VOICINGRATIO are very short, but breathing and coughing is excluded by the VOICINGRATIO threshold, as they consist of mostly unvoiced frames. 
We note that at a true positive rate (recall rate) of $90 \%$, we obtain as few as $22 \%$ false positives on the DURATION curve. Conversely, at a rate of zero false positives, we still capture $10 \%$ of the true positives on the VOICINGRATIO curve. Finally, we note that discrimination using VOICINGRATIO and DURATION appears to be complementary in part. Most importantly, placing the constraint that a talkspurt should contain a non-zero amount of voiced speech may have a positive effect on limiting false positives. The confusion matrix of one annotator's LOW/NONLOW decisions versus automatic VSU identification using a combination of VOICINGRATIO and DURATION is shown in Table 2.

Table 2. Confusion matrix of manual annotation (annotator 1) versus VSUs.

\begin{tabular}{llrrr}
\hline & & \multicolumn{3}{c}{ Automatic } \\
& & VSU & NonVSU & Total \\
\hline \multirow{3}{*}{ Rater 1 } & LOW & $\mathbf{8 5 9}$ & 205 & 1064 \\
& NONLOW & 152 & $\mathbf{1 8 3 8}$ & 1990 \\
& Total & 1011 & 2043 & $\mathbf{3 0 5 4}$ \\
\hline
\end{tabular}

\subsection{Bitmap clusters of VSUs}

In order to visualize the discrepancies between automatic VSU identification and manual LOW annotation, we produce bitmap clusters for the four cells in Table 2. These are shown in Figure 5.

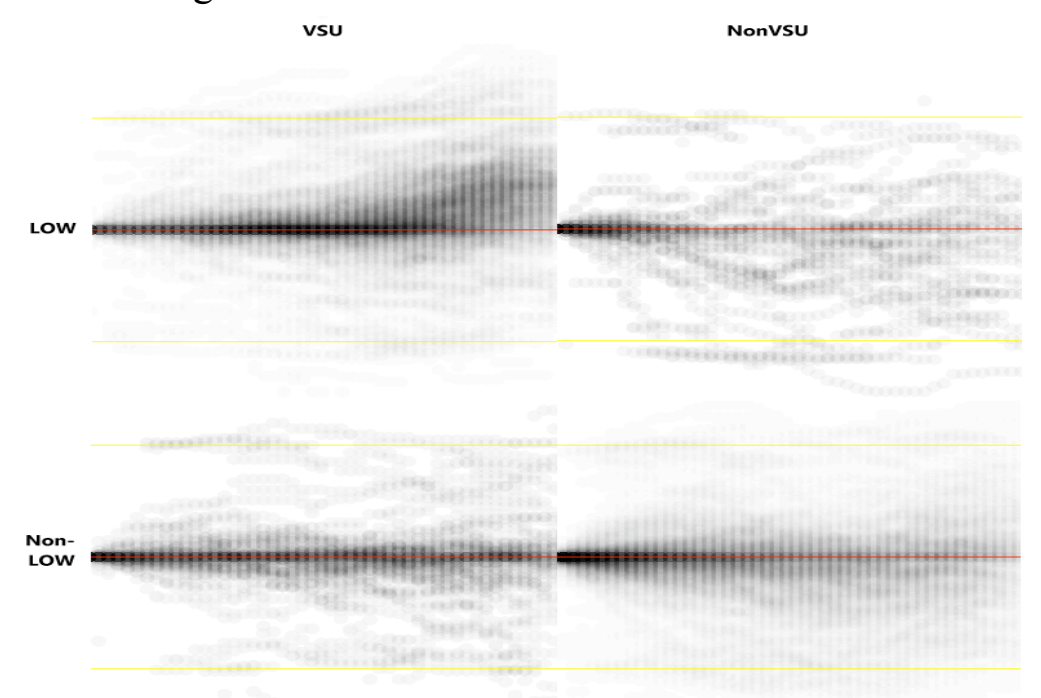

Figure 5. Bitmap clusters (see 2.2.4 above) of the final 500ms of talkspurts labeled LowVSU (upper left panel), LOW-NONVSU (upper right panel), NONLOW-VSU (lower left panel), and NONLOW-NONVSU (lower right panel). 
The bitmap clusters reveal a clear tendency for rising patterns at the end of VSUs judged as Low, whereas NonLOw NONVSUs fail to show such a rise and have most of the activity in the lower half of the panel, possibly indicating a preference for falling contours. The upper right and lower left panes, where human judgments and automatic classification are in conflict, contain much less data (cf. Table 2) and show no clear patterns. They account for roughly $12 \%$ of talkspurts, and we leave their detailed analysis for future work, when more data becomes available.

The observation of rising patterns in the LOW VSUs is in line with our recent observation (Heldner, et al., forthcoming) of a tendency for speakers in the follower role of the Map Task to end utterances in a rising tone, as the follower role contains a high proportion of backchannels.

\subsection{Bitmap clusters of VSUs preceding interlocutor speech}

Figure 6 shows bitmap clusters of the 1000ms of the interlocutor's speech preceding each of the 3054 talkspurts.

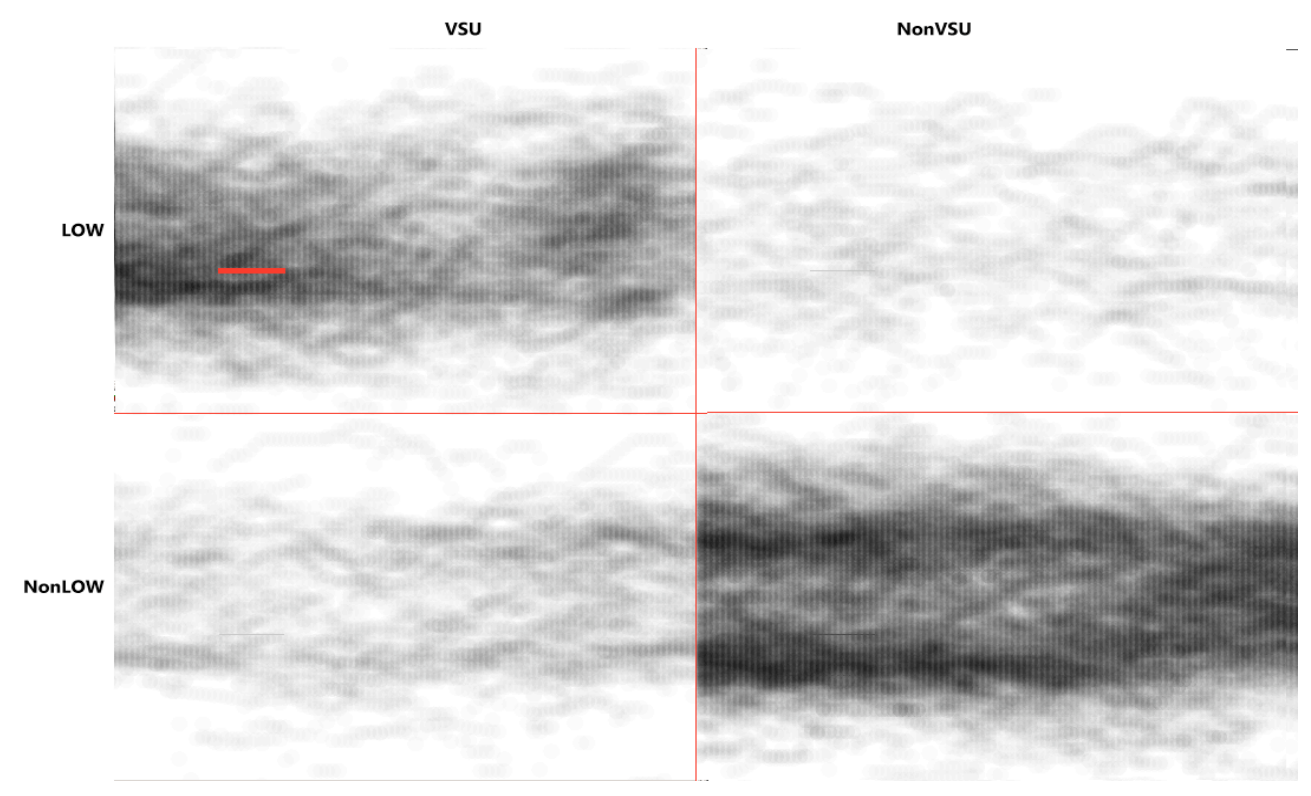

Figure 6. Plots of pitch contours as described in 2.2 .4 above, of the $1000 \mathrm{~ms}$ immediately preceding each talkspurt in the other speaker's channel. The panels show the speech preceding talkspurts labeled as LOW-VSU (upper left panel), as LOW-NONVSU (upper right panel), as NONLOW-VSU (lower left panel), and as NONLOW-NONVSU (lower right panel). The upper left panel contains a line showing the $26^{\text {th }}$ percentile of the speakers' pitch from 860 to $700 \mathrm{~ms}$ prior to the talkspurt. 
In Figure 6, we observe that interlocutor speech preceding talkspurts judged as LOW and identified as VSUs is compatible with the finding that $700-860 \mathrm{~ms}$ prior to the start of a backchannel, interlocutors exhibit increased activity below the $26^{\text {th }}$ percentile of their pitch range (Ward \& Tsukahara, 2000) and little activity in above the center line, as opposed to the lower right panel. The top left panel contains a line on the $26^{\text {th }}$ percentile along the $700-860 \mathrm{~ms}$ interval for illustration. This qualitative agreement is an indication that VSUs capture phenomena which Ward and others refer to as backchannels.

In the lower right panel, we observe a relatively symmetric, bimodal distribution. There is a somewhat lighter band along the horizontal mid-line, indicating an interlocutor dispreference for approximately mean pitch before the onset of NONLOw NONVSUs, which is in line with previous findings (cf. Edlund $\&$ Heldner, 2005, and references mentioned therein), namely that mid-level pitch is a common floor-keeper.

\section{Conclusions}

The very short utterance (or VSU) proposed in this work is automatically identified using easily accessible acoustic information such as the duration of and proportion of voicing in talkspurts. Our analyses suggest that it captures a large proportion of the dialogue phenomena commonly referred to as backchannels. This is evidenced by: the strong overlap between VSUs and manually labeled low-content talkspurts; by the similarity in final pitch contour between VSUs and the backchannel-intensive follower role in the Map Task; and by the fact that interlocutor pitch $700-860 \mathrm{~ms}$ prior to a VSU is consistent with Ward \& Tsukahara's (2000) findings regarding interlocutor pitch preceding backchannels. Prosodically, VSUs are characterized by a final rising pitch, in addition to the duration and voicing characteristics that define them.

The finding that the easily distinguishable VSUs share many characteristics with backchannels has important implications for future research. From a basic research perspective, it means that we can, at zero manual effort, make more detailed descriptions of conversational behavior. We may for example separate speaker changes involving VSUs from others. From a speech technology perspective, VSUs may be used to afford spoken dialogue systems less rigid interaction control by allowing VSUs to be interpreted differently from other user utterances. For example, if users are allowed to barge in - to speak in overlap with the system - the system may go on uninterrupted in case the user utterance is a VSU, and relinquish the floor if it is not. We believe this to be an important step towards more human-like behavior in spoken dialogue systems. 


\section{Acknowledgements}

We thank Kornel Laskowski for insightful discussions and criticism, and Pétur Helgason for access to the Swedish Map Task Corpus. Funding was provided by the Swedish Research Council (VR) project 2006-2172 Vad gör tal till samtal?

\section{References}

Brady, P. T. (1968). A statistical analysis of on-off patterns in 16 conversations. The Bell System Technical Journal, 47, 73-91.

de Cheveigné, A., \& Kawahara, H. (2002). YIN, a fundamental frequency estimator for speech and music. Journal of the Acoustical Society of America, 111(4), 1917-1930.

Edlund, J., \& Heldner, M. (2005). Exploring prosody in interaction control. Phonetica, 62(2-4), 215-226.

Hardy, H., Baker, K., Bonneau-Maynard, H., Devillers, L., Rosset, S., \& Strzalkowski, T. (2003). Semantic and dialogic annotation for automated multilingual customer service. In EUROSPEECH-2003 (pp. 201-204). Geneva, Switzerland.

Heldner, M., Edlund, J., Laskowski, K., \& Pelcé, A. (forthcoming). Prosodic features in the vicinity of silences and overlaps. In Nordic Prosody $X$ (this volume). Helsinki, Finland.

Helgason, P. (2006). SMTC - A Swedish Map Task Corpus. In Working Papers 52: Proceedings from Fonetik 2006 (pp. 57-60). Lund, Sweden.

Jurafsky, D., Shriberg, E., \& Biasca, D. (1997). Switchboard SWBD-DAMSL Annotation Coders Manual, Draft 13 Retrieved 2008-12-06, from http://www.stanford.edu/ jurafsky/ws97/manual.august1.html

Laskowski, K., Edlund, J., \& Heldner, M. (2008). An instantaneous vector representation of delta pitch for speaker-change prediction in conversational dialogue systems. In Proceedings ICASSP 2008 (pp. 5041-5044). Las Vegas, $\mathrm{NV}$, USA.

Norwine, A. C., \& Murphy, O. J. (1938). Characteristic time intervals in telephonic conversation. The Bell System Technical Journal, 17, 281-291.

Schegloff, E. (1982). Discourse as an interactional achievement: Some uses of 'uh huh' and other things that come between sentences. In D. Tannen (Ed.), Analyzing Discourse: Text and Talk (pp. 71-93). Washington, D.C., USA: Georgetown University Press.

Shriberg, E., Bates, R., Stolcke, A., Taylor, P., Jurafsky, D., Ries, K., et al. (1998). Can prosody aid in the automatic classification of dialog acts in conversational speech. Language and Speech, 41(3-4), 439-487.

Ward, N. (2004). Pragmatic functions of prosodic features in non-lexical utterances. In Proceedings of Speech Prosody 2004 (pp. 325-328). Nara, Japan.

Ward, N., \& Tsukahara, W. (2000). Prosodic features which cue back-channel responses in English and Japanese. Journal of Pragmatics, 32, 1177-1207.

Yngve, V. H. (1970). On getting a word in edgewise. In Papers from the Sixth Regional Meeting Chicago Linguistic Society (pp. 567-578). Chicago, IL, USA: Chicago Linguistic Society. 